\title{
Rosiglitazone inhibits insulin-like growth factor-1-induced polycystic kidney disease cell growth and p70S6 kinase activation
}

\author{
CHUNYAN LIU $^{1}$, YI ZHANG ${ }^{2}$, LI YUAN ${ }^{2}$, LILI FU $^{2}$ and CHANGLIN MEI ${ }^{2}$ \\ ${ }^{1}$ Department of Nephrology, The Second Affiliated Hospital of Dalian Medical University, Liaoning 116027; \\ ${ }^{2}$ Department of Nephrology, Changzheng Hospital, Second Military Medical University, Shanghai 200003, P.R. China
}

Received February 1, 2013; Accepted June 18, 2013

DOI: $10.3892 / \mathrm{mmr} .2013 .1588$

\begin{abstract}
Autosomal dominant polycystic kidney disease (ADPKD) is one of the most common genetic kidney disorders. Thiazolidinediones (TZDs) are anti-diabetic drugs that have been shown to suppress polycystic kidney diseases (PKD) development. However, their underlying mechanism of action remains largely unknown. Insulin-like growth factor-1 (IGF-1) expression increases with the progression of cystic lesions in ADPKD and murine PKD, thus the increased expression of IGF-1 may contribute to the progression of cystic lesions. p70S6 kinase (p70S6K) is an important downstream signaling molecule of IGF-1 and is implicated in the regulation of cell cycle progression and cell proliferation. In the present study, we found that IGF-1 increased the growth of cyst-lining epithelial cells by $15-20 \%$ in a dose-dependent manner, while no effect on the proliferation of normal renal cortical tubular epithelial cells (RCTEC) was observed. Rosiglitazone, a TZD, was found to inhibit the IGF-1-induced growth of cyst-lining epithelial cells when applied at a dose of 50-200 $\mu \mathrm{M}$. However, the IGF-1-induced growth of immortalized epithelial cells from $>30$ individual renal cysts obtained from 11 ADPKD patients (WT9-12 cells) was inhibited with a $12.5-\mu \mathrm{M}$ dose of rosiglitazone. Moreover, rosiglitazone (at the same concentration) was shown to inhibit the IGF-1induced activation of p70S6K. TZDs are known to exert antitumor properties via peroxisome proliferator-activated receptor (PPAR) $\gamma$-dependent and -independent mechanisms. The present study showed that PPAR $\gamma$ small interfering RNA (siRNA) did not block the effect of rosiglitazone in inhibiting the IGF-1-induced phosphorylation of p70S6K. In conclusion, cyst-lining epithelial cells were found to be more sensitive to IGF-1 compared with normal cells. Rosiglitazone inhibited the proliferation of cyst-lining epithelial cells; more specifically, it inhibited the proliferation-promoting activity of IGF-1
\end{abstract}

Correspondence to: Professor Changlin Mei, Department of Nephrology, Changzheng Hospital, Second Military Medical University, 415 Fengyang Road, Shanghai 200003, P.R. China

E-mail: chlmei@hotmail.com

Key words: autosomal dominant polycystic kidney disease, rosiglitazone, insulin-like growth factor-1, p70S6 kinase in these cells. This effect of rosiglitazone was demonstrated to be partially due to the inhibition of IGF-1-induced activation of p70S6K. Increased IGF-1 expression was identified in early-stage PKD, indicating that rosiglitazone is more suitable for the treatment of early-stage PKD.

\section{Introduction}

Autosomal dominant polycystic kidney disease (ADPKD) is one of the most common genetic disorders worldwide, with a frequency of 1 in 1,000 in the general population. The excessive proliferation of renal tubular epithelial cells leads to cysts that eventually replace most of the normal tissue. Consequently, ADPKD results in severe enlargement of the kidneys, and renal failure occurs by the age of 50 in the majority of cases (1-2).

Insulin-like growth factor-1 (IGF-1) preferentially binds to IGF-1 and insulin receptors. The two receptors are structurally similar and a number of their downstream molecules are the same, including insulin receptor substrate-1 (IRS-1), phosphoinositide 3-kinase (PI3K), protein kinase B (PKB/Akt), mammalian target of rapamycin (mTOR) and p70S6 kinase (p70S6K), while their physiological functions are different. IGF-1 signaling favors cell growth, proliferation and survival in relation to nutrient availability (3). IGF-1 has a well-documented role in cancer development in several tissues (4), and its expression has been found to increase with the progression of cystic lesions in ADPKD and murine polycystic kidney diseases (PKDs) (5); therefore, IGF-1 may contribute to the progression of cystic lesions.

Thiazolidinediones (TZDs) are anti-diabetic drugs that improve insulin sensitivity in patients with type 2 diabetes since they are high-affinity ligands for peroxisome proliferator-activated receptor (PPAR) $\gamma$, a transcription factor that is highly expressed in adipose tissues. Although TZDs are only approved for the treatment of type 2 diabetes, they are also known to have additional potentially beneficial effects (6). It has been shown that TZDs are capable of inhibiting cell proliferation and inducing apoptosis in a wide variety of tumor cell lines (7). Recent studies have suggested that TZDs inhibit the progression of PKD (8-10). However, the underlying mechanism of action of TZDs in PKD remains unknown.

The IGF-1/p70S6K pathway is activated in PKD, and rosiglitazone has been suggested to inhibit the expression of 
IGF-1. Therefore, the present study used ADPKD cyst-lining epithelial cells to investigate the effect of rosiglitazone on IGF-1 mitogenic signaling, particularly on the IGF-1-induced activation of p70S6K.

\section{Materials and methods}

Materials. Rosiglitazone was purchased from Sigma-Aldrich (St. Louis, MO, USA). Stock solution was prepared in dimethyl sulfoxide (DMSO) at $200 \mathrm{mM}$ and stored in aliquots at $-80^{\circ} \mathrm{C}$. Antibodies against p70S6K, p-p70S6K-Thr ${ }^{421 / 424}$, PPAR $\gamma$ and IGF-1 were purchased from Cell Signaling Technology, Inc. (Beverly, MA, USA).

Cell culture and treatment. Immortalized epithelial cells from $>30$ individual renal cysts obtained from 11 ADPKD patients (WT9-12) and normal human renal cortical tubular epithelial cells (RCTEC) were kindly provided by Dr Jing Zhou (Harvard Institutes of Medicine, Harvard Medical School, Boston, MA, USA) (11). The cells were maintained in Dulbecco's modified Eagle's medium (DMEM) supplemented with $10 \%$ fetal bovine serum (FBS) and $100 \mathrm{U} / \mathrm{ml}$ each of penicillin and streptomycin. The cells were seeded at a density of 4,000 cells/well in a 96-well plate. After $24 \mathrm{~h}, 10 \%$ FBS growth medium was replaced with serum-free medium. Cells were either treated with rosiglitazone or IGF-1, or the combination of both. Cell growth curves were determined 24,48 and $72 \mathrm{~h}$ following treatment using a tetrazolium salt 3-(4,5-dimethylthiazol-2-yl)-2,5-diphenyltetrazolium bromide (MTT) assay. Briefly, MTT $(500 \mu \mathrm{g} / \mathrm{ml})$ was added to cells for $4 \mathrm{~h}$. The cells were then collected using a DMSO solution. The rate of MTT uptake and the formation of blue formazan crystals by live cells were determined by absorption at a wavelength of $490 \mathrm{~nm}$ using an enzyme-linked immunosorbent assay (ELISA) reader (ELX 800; BioTek Intstruments, Winooski, VT, USA).

Western blot analysis. For western blot analysis, the cells treated with various chemicals were washed with ice-cold phosphate-buffered saline (PBS) and lysed in RIPA buffer (1 mM phenylmethylsulfonyl fluoride, $10 \mathrm{mM} \beta$-glycerophosphate, $1 \mathrm{mM} \mathrm{NaF}$ and $1 \mathrm{mM} \mathrm{Na}_{3} \mathrm{VO}_{4}$ ) containing protease inhibitor cocktail. Protein concentrations were determined using a BCA protein assay kit (Pierce Biotechnology, Inc., Rockford, IL, USA). Equal amounts of protein (100 $\mu \mathrm{g})$ were loaded and separated on SDS-polyacrylamide gels and then transferred to nitrocellulose membranes. After blocking, membranes were probed with antibodies against p70S6K, p-p70S6K-Thr ${ }^{421 / 424}$ and PPAR $\gamma$ followed by the corresponding horseradish peroxidase (HRP)-conjugated secondary antibody. Positive staining was revealed via a reaction with an enhanced chemiluminescence (ECL) reagent and development on an X-ray film.

Transfection with small interfering RNAs (siRNAs). PPAR $\gamma$ and non-specific control siRNAs were purchased from Cell Signaling Technology, Inc. For the transfection, the cells were grown until 30-40\% confluence, and PPAR $\gamma$ or non-specific control siRNAs were introduced into the cells using the Lipofectamine $^{\mathrm{TM}} 2000$ reagent (Invitrogen, Carlsbad, CA, CA) according to the manufacturer's instructions. Briefly,

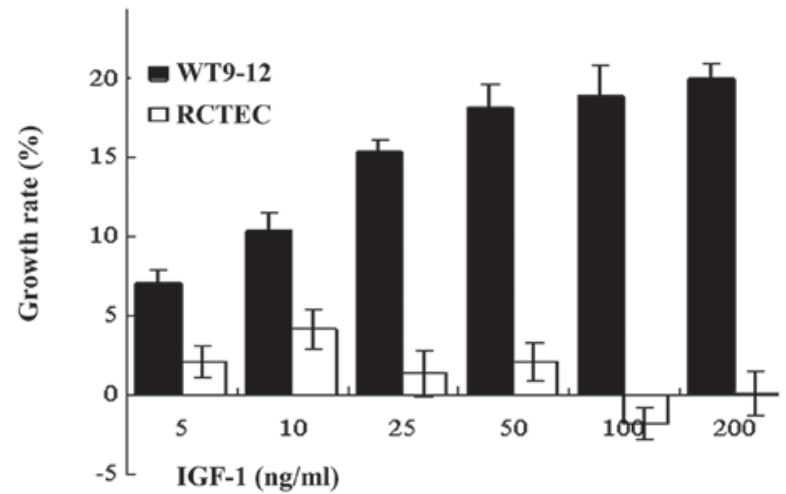

Figure 1. IGF-1 stimulates WT9-12 cell, but not normal human renal cortical tubular epithelial cell (RCTEC) proliferation. WT9-12 and RCTEC cells were incubated with IGF-1 at the indicated concentrations for $72 \mathrm{~h}$. The rate of cell growth was determined $72 \mathrm{~h}$ following treatment using an MTT assay. Results shown are the averages of three different experiments. IGF-1, insulinlike growth factor-1.

Lipofectamine reagent and siRNA were diluted in a serum-free medium. After $5 \mathrm{~min}$, the two solutions were mixed and placed at room temperature for an additional $20 \mathrm{~min}$. The mixture was then added to the cells. The final concentration of siRNA in each well was $100 \mathrm{nM}$. A number of cells were treated with rosiglitazone $(50 \mu \mathrm{M}) 48 \mathrm{~h}$ after the transfection, and then cell lysates were collected for western blot analysis $24 \mathrm{~h}$ after this treatment.

Statistical analysis. All the experiments were repeated at least three times. Data were expressed as the mean \pm SD. Statistical significance was determined with Student's t-test (two-tailed) for comparisons between two groups. $\mathrm{P}<0.05$ was considered to indicate a statistically significant difference.

\section{Results}

IGF-1 increases the proliferation of cyst-lining epithelial cells. The growth of the cyst-lining epithelial cell line WT9-12 was compared with that of a normal cell line (RCTEC) to investigate the effect of IGF-1 on cell proliferation. The effect of IGF-1 on cell growth was examined by treating the cells with various concentrations of IGF-1 $(5-200 \mathrm{ng} / \mathrm{ml})$. The rate of cell growth was determined $72 \mathrm{~h}$ following treatment using an MTT assay. IGF-1 treatment was found to increase WT9-12 cell growth by $15-20 \%$ in a dose-dependent manner, while it had no effect on RCTEC cell proliferation (Fig. 1).

Rosiglitazone inhibits IGF-1-induced PKD cell growth. The effect of rosiglitazone or rosiglitazone combined with IGF-1 on cell growth was examined in order to investigate the effect of rosiglitazone on IGF-1-induced PKD cell growth. Rosiglitazone at doses of 50-200 $\mu \mathrm{M}$ was found to inhibit WT9-12 cell proliferation in a dose-dependent manner (Fig. 2). However, IGF-1-induced WT9-12 cell proliferation was inhibited with a $12.5-\mu \mathrm{M}$ dose of rosiglitazone (Fig. 3).

Rosiglitazone inhibits IGF-1-induced phosphorylation of p70S6K in a PPAR $\gamma$-independent manner. The p70S6K signaling molecule is involved in the regulation of cell cycle 


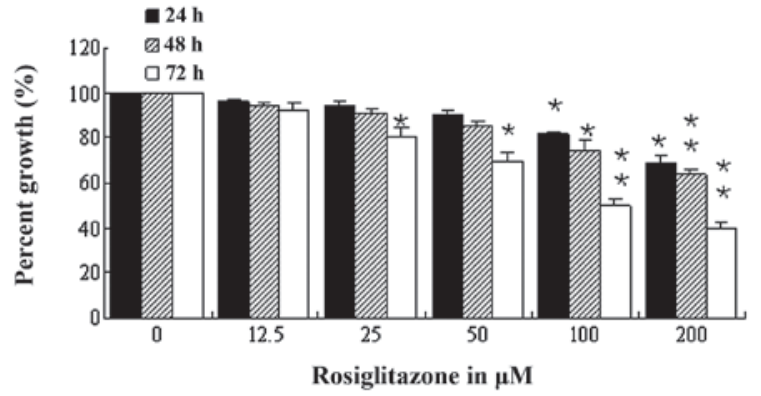

Figure 2. Rosiglitazone inhibits the proliferation of the autosomal dominant polycystic kidney disease (ADPKD) cyst-lining epithelial cell line WT9-12 in a dose-dependent manner. WT9-12 cells were incubated with rosiglitazone at the indicated concentrations for $72 \mathrm{~h}$. The rate of cell growth was determined $72 \mathrm{~h}$ following treatment using an MTT assay. Results shown are the averages of three different experiments. ${ }^{*} \mathrm{P}<0.05$ and ${ }^{* *} \mathrm{P}<0.01$ compared with control cells. IGF-1, insulin-like growth factor-1.

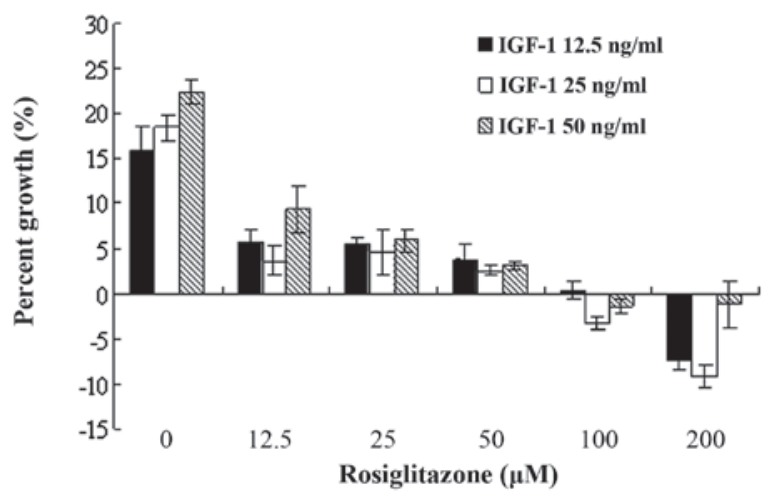

Figure 3. Rosiglitazone inhibits IGF-1-induced proliferation of WT9-12 cells. WT9-12 cells were initially incubated with IGF-1 at various concentrations for $0.5 \mathrm{~h}$, followed by incubation in culture medium supplemented with rosiglitazone at various concentrations for $72 \mathrm{~h}$. The rate of cell growth was determined $72 \mathrm{~h}$ following treatment using an MTT assay. Results shown are the averages of three different experiments.IGF-1, insulin-like growth factor-1.

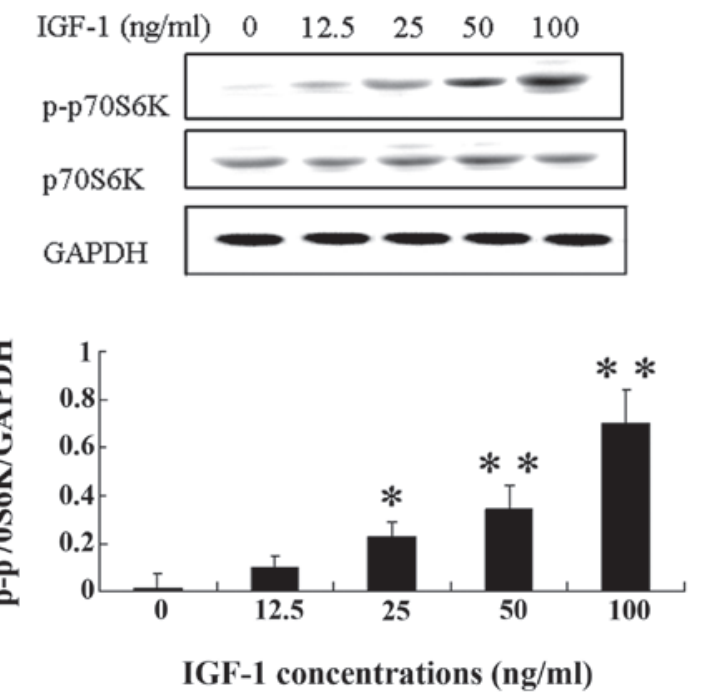

Figure 4. IGF-1 induces the phosphorylation of p70S6K in a dose-dependent manner. WT9-12 cells were cultured with increasing concentrations of IGF-1 for $24 \mathrm{~h}$. Control cells were treated with $0.05 \%$ Dulbecco's modified Eagle's medium (DMSO) alone. Methods of western blot analysis using the specific antibodies are described in Materials and methods. ${ }^{*} \mathrm{P}<0.05$ and ${ }^{* *} \mathrm{P}<0.01$ compared with the control cells. IGF-1, insulin-like growth factor-1; p70S6K, p70S6 kinase; p-p70S6K, phosphorylated p70S6K.
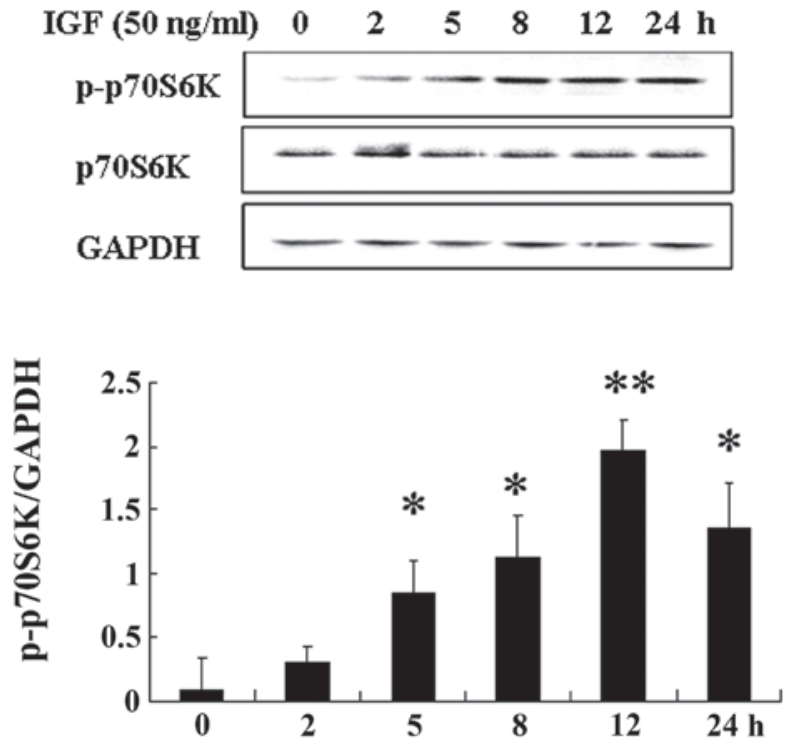

Figure 5. IGF-1 $(20 \mathrm{ng} / \mathrm{ml})$ induces the phosphorylation of p70S6K in a time-dependent manner. WT9-12 cells were cultured with $50 \mathrm{ng} / \mathrm{ml} \mathrm{IGF-1} \mathrm{for}$ the indicated times. Control cells were treated with $0.05 \%$ Dulbecco's modified Eagle's medium (DMSO) alone. Methods of western blot analysis using the specific antibodies are described in Materials and methods. ${ }^{*} \mathrm{P}<0.05$ and ${ }^{* *} \mathrm{P}<0.01$ compared with the control cells. IGF-1, insulin-like growth factor-1; p70S6K, p70S6 kinase; p-p70S6K, phosphorylated p70S6K.
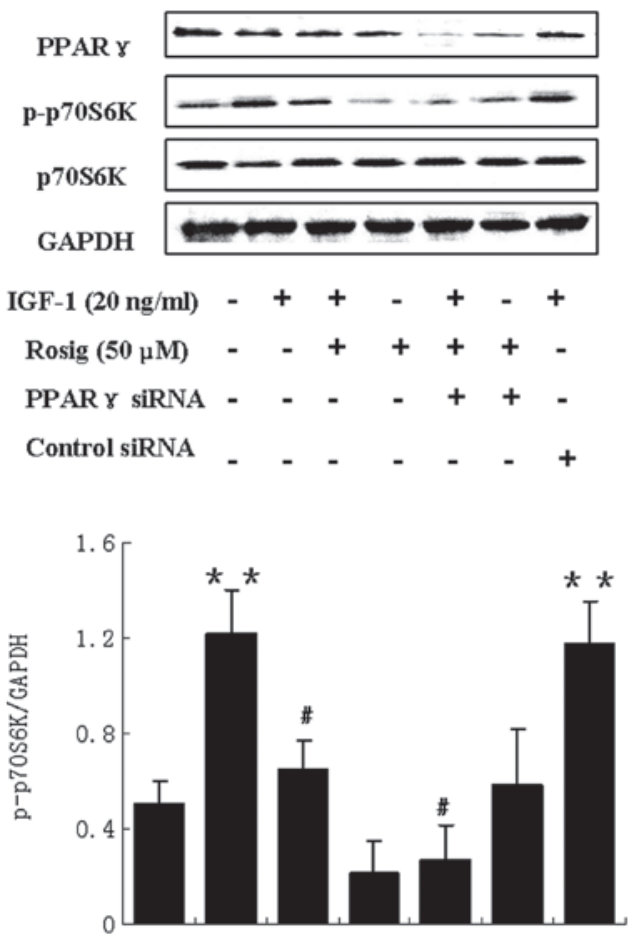

Figure 6. Rosiglitazone inhibits the IGF-1-induced phosphorylation of p70S6K through a PPAR $\gamma$-independent pathway. WT9-12 cells were transfected with PPAR $\gamma$ or control siRNA. The cells were then treated with IGF-1 $(20 \mathrm{ng} / \mathrm{ml})$ or rosiglitazone $(50 \mu \mathrm{M})$ or the combination of both for $12 \mathrm{~h}$. Control cells were treated with $0.05 \%$ Dulbecco's modified Eagle's medium (DMSO) alone. Cell lysates were then collected for western blot analysis. Methods of western blot analysis using the specific antibodies are described in Materials and methods. ${ }^{* *} \mathrm{P}<0.01$ compared with the control cells; ${ }^{~} \mathrm{P}<0.05$ compared with the cells treated with IGF-1 alone. Rosig, rosiglitazone; IGF-1, insulin-like growth factor-1; p70S6K, p70S6 kinase; p-p70S6K, phosphorylated $\mathrm{p} 70 \mathrm{~S} 6 \mathrm{~K}$; PPAR $\gamma$, peroxisome proliferator-activated receptor $\gamma$; siRNA, short interfering RNA. 
progression and cell proliferation (12). We found that IGF-1 increased the phosphorylation of p70S6K in a dose- and time-dependent manner in WT9-12 cells (Figs. 4 and 5). The expression of p-P70S6K was increased after WT9-12 cells were treated with IGF-1 (20 ng/ml) for $2 \mathrm{~h}$.

Rosiglitazone was found to inhibit IGF-1-induced phosphorylation of p70S6K (Fig. 6). Rosiglitazone, a synthetic ligand for PPAR $\gamma$, inhibits cell growth through PPAR $\gamma$-dependent and -independent signaling pathways (19). Therefore, we investigated whether the effect of rosiglitazone on IGF-1-induced phosphorylation of p70S6K was mediated by the activation of PPAR $\gamma$. WT9-12 cells were transfected with PPAR $\gamma$ or control siRNA. The cells were then treated with IGF-1 or rosiglitazone, or the combination of both. As shown in Fig. 6, the effect of rosiglitazone on IGF-1-induced p70S6K phosphorylation was unable to be blocked. This suggests that rosiglitazone may inhibit IGF-1-induced phosphorylation of p70S6K in a PPAR $\gamma$-independent manner.

\section{Discussion}

One of the main changes in the PKD kidney is an increase in the proliferative activity of cyst-lining epithelial cells. Proliferating cells are frequently detected in normal tubular segments and are relatively sparse in large cysts. These findings suggest that the increase in proliferative activity is an early event and may predispose these cells to the acquisition of a cystic phenotype $(13,14)$.

IGF-1 is a multifunctional hormone that has pleiotropic effects on cellular proliferation, apoptosis, hypertrophy, senescence and differentiation. Multiple lines of evidence suggest that IGF-1 plays a role in mediating tubular cell proliferation in the cystic kidney, particularly during the early stages of PKD. The present and previous studies have found that IGF-1 induces PKD cell proliferation, while no effect has been observed in normal tubular cells (15). This indicates that PKD cells are more sensitive to IGF-1 compared with normal cells.

Rosiglitazone is effective in regulating cell activation, differentiation, proliferation and/or apoptosis (16). The efficacy of this compound as an anticancer agent has been examined in a variety of cancers, including colon, breast, prostate and non-small cell lung carcinoma $(6,17)$. In polycystic renal disease, rosiglitazone has been suggested to inhibit the progression of PKD (8-18). In the present study, we showed that rosiglitazone inhibited the proliferation of PKD cells and that it had a more predominant inhibiting effect on IGF-1-induced cell proliferation. This finding indicates that rosiglitazone is suitable for the treatment of early-stage PKD.

p70S6K is an important downstream signaling molecule of IGF-1. The activity of p70S6K, which is determined by the phosphorylation of p70S6K, is enhanced in the kidneys of ADPKD patients and is important in the pathogenesis of ADPKD (19). In the present study, we found that IGF-1 induced the phosphorylation of p70S6K in PKD cells, while rosiglitazone inhibited the effect of IGF-1 on p70S6K phosphorylation. TZDs are known to act in a PPAR $\gamma$-dependent and -independent manner (19). Our study indicated that rosiglitazone inhibited IGF-1-induced phosphorylation of p70S6K through a PPAR $\gamma$-independent manner.
In summary, we found that rosiglitazone inhibited the IGF-1-induced activation of p70S6K via a PPAR $\gamma$-independent mechanism. This appears to account for, at least in part, the mechanism by which rosiglitazone inhibits IGF-1-induced PKD cell proliferation. IGF-1 has an important effect on early-stage PKD; therefore, rosiglitazone is suggested to be more effective in the treatment of early-stage PKD.

\section{Acknowledgements}

This study was supported by the National 973 Program of China (2007CB507400).

\section{References}

1. Torres VE, Harris PC and Pirson Y: Autosomal dominant polycystic kidney disease. Lancet 369: 1287-1301, 2007.

2. Kazancioglu R, Ecder T, Altintepe L, Altiparmak MR, Tuglular S, Uyanik A, et al: Demographic and clinical characteristics of patients with autosomal dominant polycystic kidney disease: a multicenter experience. Nephron Clin Pract 117: C270-C275, 2011.

3. Nakae J, Kido Y and Accili D: Distinct and overlapping functions of insulin and IGF-I receptors. Endocr Rev 22: 818-835, 2001.

4. Pollak MN, Schernhammer ES and Hankinson SE: Insulin-like growth factors and neoplasia. Nat Rev Cancer 4: 505-518, 2004.

5. Nakamura T, Ebihara I, Nagaoka I, Tomino Y, Nagao S, Takahashi $\mathrm{H}$ and Koide $\mathrm{H}$ : Growth factor gene expression in kidney of murine polycystic kidney disease. J Am Soc Nephrol 3: 1378-1386, 1993.

6. Grommes C, Landreth GE and Heneka MT: Antineoplastic effects of peroxisome proliferator-activated receptor gamma agonists. Lancet Oncol 5: 419-429, 2004.

7. Yki-Järvinen H: Thiazolidinediones. N Engl J Med 351: 1106-1118, 2004

8. Muto S, Aiba A, Saito Y, et al: Pioglitazone improves the phenotype and molecular defects of a targeted Pkd1 mutant. Hum Mol Genet 11: 1731-1742, 2002.

9. Dai B, Liu Y, Mei C, et al: Rosiglitazone attenuates development of polycystic kidney disease and prolongs survival in Han: SPRD rats. Clin Sci (Lond) 119: 323-333, 2010.

10. Blazer-Yost BL, Haydon J, Eggleston-Gulyas T, Chen JH, Wang X, Gattone V and Torres VE: Pioglitazone attenuates cystic burden in the PCK rodent model of polycystic kidney disease. PPAR Res 2010: 274376, 2010.

11. Loghman-Adham M, Nauli SM, Soto CE, Kariuki B and Zhou J: Immortalized epithelial cells from human autosomal dominant polycystic kidney cysts. Am J Physiol Renal Physiol 285: F397-F412, 2003.

12. Zhou $\mathrm{H}$ and Huang $\mathrm{S}$ : The complexes of mammalian target of rapamycin. Curr Protein Pept Sci 11: 409-424, 2010.

13. Torres VE and Harris PC: Autosomal dominant polycystic kidney disease: the last 3 years. Kidney Int 76: 149-168, 2009.

14. Patel V, Chowdhury R and Igarashi P: Advances in the pathogenesis and treatment of polycystic kidney disease. Curr Opin Nephrol Hypertens 18: 99-106, 2009.

15. Parker E, Newby LJ, Sharpe CC, et al: Hyperproliferation of PKD1 cystic cells is induced by insulin-like growth factor-1 activation of the Ras/Raf signalling system. Kidney Int 72: 157-165, 2007.

16. Zieleniak A, Wójcik M and Woźniak LA: Structure and physiological functions of the human peroxisome proliferator-activated receptor gamma. Arch Immunol Ther Exp (Warsz) 56: 331-345, 2008.

17. Michalik L, Desvergne B and Wahli W: Peroxisome-proliferatoractivated receptors and cancers: complex stories. Nat Rev Cancer 4: 61-70, 2004.

18. Shillingford JM, Murcia NS, Larson $\mathrm{CH}$, et al: The mTOR pathway is regulated by polycystin-1, and its inhibition reverses renal cystogenesis in polycystic kidney disease. Proc Natl Acad Sci USA 103: 5466-5471, 2006.

19. Han SW and Roman J: Rosiglitazone suppresses human lung carcinoma cell growth through PPARgamma-dependent and PPARgamma-independent signal pathways. Mol Cancer Ther 5: 430-437, 2006. 